\title{
14
}

\section{Direct-Manipulation Spatial Exploration Using SVIQUEL}

Sudhir Kaushik and Elke A. Rundensteiner

Worcester Polytechnic Institute

Department of Computer Science

100 Institute Road, Worcester, MA 01609

sudhir@cs.wpi.edu, rundenst@cs.wpi.edu

\section{Abstract}

This paper represents the first effort to apply direct-manipulation (VIS) techniques to spatial data exploration providing mechanisms for visually querying as well as browsing spatial data and for reviewing the visual results for trend discovery. We present a visual query language (SVIQUEL) which allows us to specify the relative spatial position (both topology and direction) between objects using direct manipulation of spatial query filters. The SVIQUEL sliders (S-sliders) are complimented by an Active-Picture-for-Querying (APIQ) providing qualitative visual representations of the quantitative query specified by the S-sliders. This increases the utility of the system for spatial browsing and spatial trend discovery with no particular query in mind. The tight coupling between the quantitative S-sliders and qualitative APIQ allows the users to work either qualitatively or quantitatively depending on his/her particular needs and freely switch between the two while working in a continuous data exploration mode.

\section{Keywords}

Direct-manipulation, spatial visual query language, integrated neighborhood model, spatial trend discovery, tight coupling.

\section{INTRODUCTION}

The exploration of large information spaces remains a challenging task especially with the growth of the World Wide Web and other such huge repositories [AS94]. Many users are not able to cope with the flood of information. Visual Information Seeking (VIS) addresses this problem by recognizing the enormous capacity of human visual information processing [AS94]. By presenting information visually and allowing user interaction through directmanipulation, users can traverse larger information spaces in shorter time [Shn92]. Our goal is to apply the concept of VIS to special-purpose data types such as spatial and temporal data, as to the best of our knowledge VIS has up to now only been applied to simple numeric data types [AS94] [WS92].

Our previous work focussed on designing a direct manipulation environment for one-dimensional temporal data, called TVQL [HR97] [HR96a] [HR96b] [HR98a], that allowed a user to visually specify temporal queries over video data giving an instantaneous visualization of the results of the query. Formal 
user studies of TVQL show that users could more accurately specify and more quickly adjust queries using TVQL than using forms [HR97] [HR98a], indicating the promise of our approach.

In this paper, we study the extension of the above concepts for two-dimensional spatial data. In particular, our contributions are:

- An integrated framework for spatial data analysis incorporating both direction and topology queries into one uniform exploration environment.

- A visual query interface for spatial data (SVIQUEL) over this integration of direction and topology expanding VIS to more complex data types (spatial in this case) which has not been attempted before.

- An active picture (APIQ) for query specification at the qualitative level and for visual query disambiguation of the quantitative S-sliders.

- Mapping functions between quantitative settings of S-sliders to qualitative query semantics in APIQ, providing for a tight coupling between the two.

- A working implementation of SVIQUEL implemented using the AWT library of JDK1.1.3 (http://metal.wpi.edu/mmvis/mmvis.html).

Section 2 reviews related work. Section 3 gives an overview of our approach. The SVIQUEL slider interface and APIQ are introduced in Section 4. Section 5 concludes the paper.

\section{RELATED WORK}

Surprisingly little has been done thus far in applying the direct manipulation paradigm to spatial environments. Visual query environments based on VIS [Shn92] [WS92] are typically restricted to simple numeric domains. The Dynamic Homefinder, a Real Estate Information Exploration System based on VIS [WS92], allows users to search for a house that meets their criteria by manipulating sliders [AS94] for different input parameters (such as number of rooms, price, distance from a fixed point, etc). However, it does not allow the user to specify the relative spatial position of two objects. Our goal is to provide a new class of dynamic filters tuned for continuous spatial querying.

Most existing spatial query languages are textual (SQL-based) [Ege94]. The possibly most popular GIS system MapInfo * uses an SQL-style language to specify queries to query primarily over non-spatial and a few spatial attributes such as area and distance of the underlying data. This language however is neither incremental nor direct manipulation based and thus our proposed interface is complimentary to such technology.

Cigales [WCL ${ }^{+}$94] is an example of a visual spatial query language, based on the Query by Example paradigm, that specifies the query using an actual drawing. GEO-QUEL [BS77] and Query by Pictorial Example [CF80] are examples of query languages where an image depicting the relationship is drawn as a query to the system.

"More information about MapInfo can be found at "http://www.mapinfo.com" 


\section{THE SPATIAL EXPLORATION ENVIRONMENT}

The four major components of our system include the spatial slider interface (S-sliders), the Active Picture for Querying interface (APIQ), the Spatial Query Processor and the Spatial Visualization of results (SVIZ). A user specifies a query using a combination of the S-sliders and APIQ interfaces. The S-slider interface is tightly coupled to APIQ allowing both qualitative and quantitative spatial data exploration. APIQ also presents a valuable aid in confirming the query specified in a visually intuitive manner. The quantitative queries expressed by the S-sliders are mapped to range queries to be processed by the query processor [HR96b, HR98b]. Once processed against the spatial database, a graphical visualization of the results is presented to the user via SVIZ. The visualization is immediately updated whenever users manipulate one of the interfaces. Thus, users can gain a sense of causality between adjusting a query interface and the visualization of the results.

\section{THE SVIQUEL INTERFACE}

Application Example. Assume a real estate system where users browse for houses that can have different spatial positions relative to the lakes and parks in the region. A user interested in finding houses that are near a lake would want to browse over the different relative positions that the houses occupy with respect to the lakes until he locates a suitable house.

\subsection{S-sliders: Spatial Dynamic Query Filters}

Our goal is to allow for the direct-manipulation of relative spatial position relationships that capture both topology and direction between two sets of spatial objects. Examples of such relationships between two objects A and B are depicted in Figure 1, with each primitive spatial relationship being called a SPRIM. Each SPRIM can be fully characterized by specifying the left and

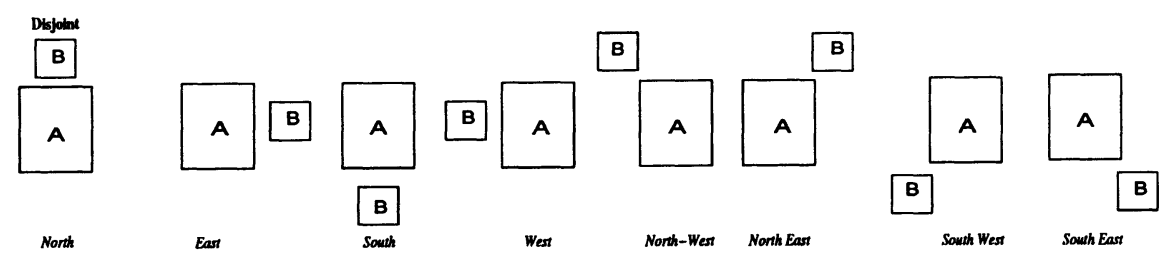

Figure 1 Spatial Primitives (SPRIMs) for the Disjoint Topology.

right ends in the $\mathrm{X}$ dimension and the top and bottom ends in the $\mathrm{Y}$ dimension of A and B. A SPRIM between A and B is defined by a conjunction of the eight pairwise relationships between the extremities in each dimension such as (Aip $\theta$ Biq) where A, B denote objects A and B, i denotes either the $\mathrm{X}$ 
or the $\mathrm{Y}$ dimension, $\theta \in\{<\rangle,,=\}$ and $p, q \in\{$ left, right, top, bottom $\} .{ }^{*}$ For example, $\mathrm{Axl}<\mathrm{Bxl}$ indicates that the left end of $\mathrm{A}$, denoted by $\mathrm{Axl}$, has to be to the left ("<") of the left end of B, denoted by Bxl.

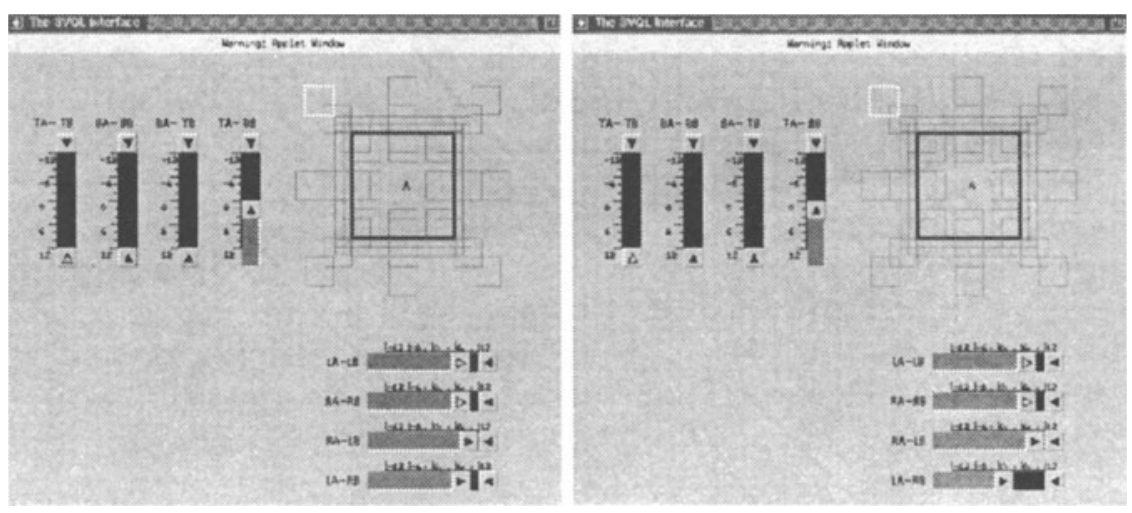

Figure 2 S-slider Query for "Houses Figure 3 S-slider Query for "Houses disjoint from and to north-west of the disjoint from and to north-west of the lake and at a distance of at least 10 lake and at a distance of at least 2 miles from lake" miles from lake"

We use eight dynamic query sliders (S-sliders) to represent these eight possible relationships as part of our SVIQUEL (see Figure 2). These eight are split up as four along each dimension, as each of the two 2D objects has four endpoint relationships along each dimension. Descriptive labels are provided alongside the sliders so that the user is able to interpret the sliders better. For example, the label LA-LB along the top-most slider indicates a continuous range of values for the difference (Left A - Left B).

Suppose the user wants to find "houses that are disjoint and to the northwest of the lake and at a distance of at least 10 miles from the lake". Figure 2 shows the positions of the S-sliders for the above query. Assume that there are only few houses this far from the lake and the user is not satisfied with the result. The user now looks for houses that are closer to the lake (say 2 miles) but still at the same relative spatial position to the lake by the simple movement of one slider. Figure 3 shows the positions of the sliders for the refined query. In order to prevent the specification of illegal queries, constraints and dependencies are introduced between the sliders so that only legal combinations of values can be specified using our interface [KR97].

${ }^{*} \mathrm{Axl}, \mathrm{Axr}, \mathrm{Bxl}, \mathrm{Bxr}$ are the extremities of $\mathrm{A}$ and $\mathrm{B}$ in the $\mathrm{X}$ dimension. Ayt, Ayb, Byt, $B y b$ are the extremities of $A$ and $B$ along the $Y$ dimension. We assume that each object has a finite non-zero width and height. The sizes of $A$ and $B$ do not convey any information about the size of the actual objects that they represent 


\subsection{APIQ: An Active Picture for Qualitative Querying}

Borrowing the idea of visual query disambiguation [HR96b], we now propose to add an Active Picture for Querying (APIQ) to SVIQUEL to provide visual qualitative representations of the query. APIQ is a natural compliment to the SVIQUEL query model as it explicitly represents all the primitive positions the houses could occupy with respect to the lake - each position graphically displayed by its SPRIM description (see top right of Figure 3 ). Once a qualitative query is specified using APIQ, then the user could refine it incrementally with quantitative constraints using S-sliders.
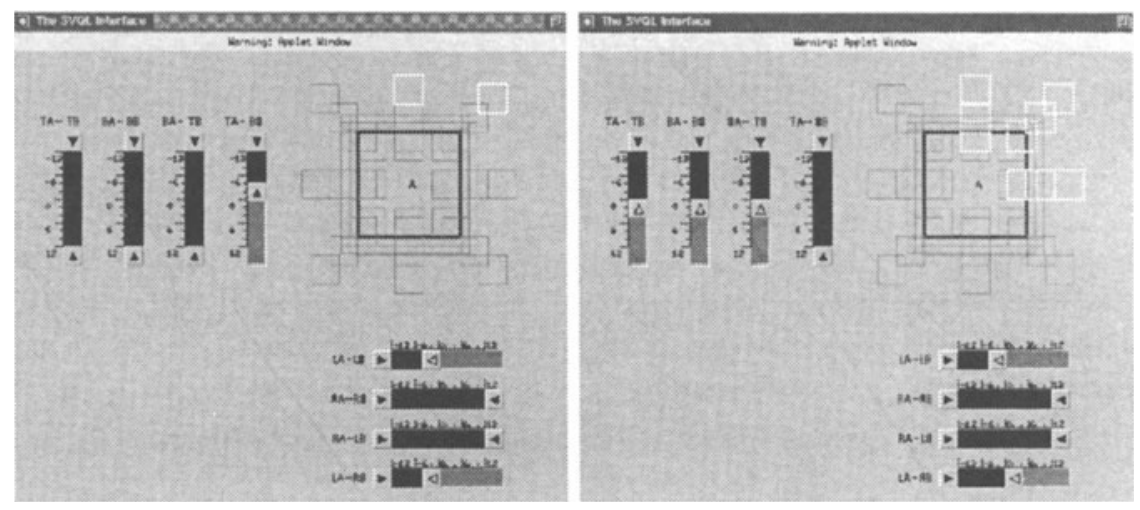

Figure 4 APIQ Query "Houses dis- Figure 5 APIQ Query "Houses disjoint and to north or north-east of joint and to north, north-east or east lake" of lake"

In APIQ, the user specifies and refines queries by selecting and de-selecting SPRIMs via mouse clicks. For example, given the APIQ in Figure 4, if the user wishes to specify the primitive disjoint east as part of the query, then a mouse click on that primitive results in including that primitive as part of the query. The tight coupling between S-sliders and APIQ achieved by our mapping functions gives new settings of the S-sliders as a result of the direct manipulation of APIQ [KR97]. The resulting APIQ and S-sliders [KR97], consistent with the integrated neighborhood model, are shown in Figure 5.

As demonstrated, APIQ increases the utility of SVIQUEL for spatial browsing with no particular query in mind. That is, both APIQ and the visualization of the results (SVIZ) are dynamically updated as users manipulate the sliders, thereby enabling the users to simply slide the sliders back and forth until an interesting result appears and then see from APIQ what query was specified.

\section{CONCLUSIONS}

In this paper, we presented the first direct-manipulation paradigm for spatial data exploration, called SVIQUEL. SVIQUEL provides users with a visual paradigm for browsing the spatial data through direct manipulation and in a 
spatially continuous manner.

Acknowledgments: We thank Stacie Hibino for developing TVQL and the Database Systems Research Group at WPI for their feedback.

\section{REFERENCES}

[AS94] C. Ahlberg and B. Shneiderman. Visual information seeking: Tight coupling of dynamic query filters with starfield displays. CHI'94, pages 313-317, Apr 1994.

[BS77] R. Berman and M. Stonebraker. Geo-Quel, a system for the manipulation and display of geometric data. ACM Computer Graphics, 11(2):186-191, 1977.

[CF80] N. S. Chang and K. S. Fu. Query by pictorial example. IEEE Trans. on Software Engg, 6(6):519-524, Nov 1980.

[Ege94] M. Egenhofer. Spatial SQL: A query and presentation language. IEEE Trans. on Knowledge and Data Eng., 6:86-95, Feb 1994.

[HR96a] S. Hibino and E. A. Rundensteiner. A visual multimedia query language for temporal analysis of video data. In MultiMedia Database Systems, pages 123-159. Kluwer Aca, 1996.

[HR96b] S. Hibino and E.A. Rundensteiner. MMVIS: Design and implementation of a multimedia VIS environment. ACM Multimedia, pages 75-86, 1996.

[HR97] S. Hibino and E. A. Rundensteiner. User study evaluation of direct-manipulation temporal interfaces. ACM Multimedia, pages 99-109, Nov 1997.

[HR98a] S. Hibino and E. A. Rundensteiner. Comparing MMVIS to a timeline for temporal trend analysis of video data. Advanced Visual Interfaces, May 1998.

[HR98b] S. Hibino and E. A. Rundensteiner. Processing incremental multidim. range queries in a direct manipulation paradigms. IEEE Int. Conf. on Data Eng., pages 458-465, Feb 1998.

[KR97] S. Kaushik and E. A. Rundensteiner. SVIQUEL: A spatial visual query and exploration language. Technical Report CS-TR-9710, Comp. Science Dept, Worcester Polytechnic Institute, 1997.

[Shn92] B. Shneiderman. Designing the User Interface: Strategies for Effective Human Computer Interaction: Second Edition. Addison Wesley Publ. Co, Reading, MA, 1992.

[WCL ${ }^{+94}$ ] A. B. Wansek, D. Calcinelli, B. Languou, C. Lecocq, and M. Mainguenaud. CIGALES: A visual query language for GIS: The user interface. International Journal of Visual Languages and Computing, 5:113-132, 1994.

[WS92] C. Williamson and B. Shneiderman. The Dynamic HomeFinder: Evaluating dynamic queries in a real-estate information exploration system. Technical Report CS-TR2819, Dept. of Computer Science,Univ. of Maryland, Jan 1992. 


\section{Biographies of Authors}

Sudhir Kaushik received a BS degree from Victoria Jubilee Technical Institute (VJTI), Bombay, India and is currently pursuing an MS degree from the Worcester Polytechnic Institute (WPI). He is a research assistant in the Department of Computer Science. His major areas of research are multimedia databases, spatial databases and web-database applications.

Elke Angelika Rundensteiner received a BS degree (Vordiplom) from the Johann Wolfgang Goethe University, Frankfurt, West Germany, an M.S. degree from Florida State University, and a Ph.D. degree from the University of California, Irvine. Dr. Rundensteiner has received numerous honors and awards, including a Fulbright Scholarship, an NSF Young Investigator Award, and an Intel Young Investigator Engineering Award, and an IBM Parnership Award. Dr. Rundensteiner is member of IEEE and ACM.

She is currently Associate professor of the Department of Computer Science at the Worcester Polytechnic Institute, after having been a faculty member in the Department of Electrical Engineering and Computer Science at the University of Michigan. Dr. Rundensteiner's focus is on developing database technology to address modeling and querying requirements of advanced applications. Her current research efforts include object-oriented databases, view techniques for data warehousing and database evolution, multi-media databases, distributed and web-database applications. 